\title{
L'Achac et la transmission du passé colonial : stratégies entrepreneuriales et culturalisation de la question immigrée dans la mémoire nationale
}

The ACHAC and the Transmission of the Colonial Past: Entrepreneurial Strategies and the Culturalization of the Immigrant Issue in the National Memory

Laurence De Cock

\section{(2) OpenEdition \\ Journals}

Édition électronique

URL : http://journals.openedition.org/conflits/19563

DOI : 10.4000/conflits. 19563

ISSN : $1777-5345$

Éditeur :

CCLS - Centre d'études sur les conflits lilberté et sécurité, L'Harmattan

Édition imprimée

Date de publication : 30 octobre 2017

Pagination : 105-121

ISBN : 978-2-343-13540-3

ISSN : 1157-996X

Référence électronique

Laurence De Cock, «L'Achac et la transmission du passé colonial : stratégies entrepreneuriales et culturalisation de la question immigrée dans la mémoire nationale », Cultures \& Conflits [En ligne], 107 | automne 2017, mis en ligne le 30 octobre 2019, consulté le 30 mars 2021. URL : http:// journals.openedition.org/conflits/19563 ; DOI : https://doi.org/10.4000/conflits.19563 


\title{
L'Achac
}

et la transmission du passé colonial : stratégies entrepreneuriales et culturalisation de la question immigrée dans la mémoire nationale

\section{Laurence DE COCK}

Laurence De Cock (ECP, Université Lyon 2) est professeure agrégée en lycée à Paris et Docteure en Sciences de l'éducation. Elle est l'auteure de nombreux articles sur l'enseignement de l'histoire. Ses travaux portent sur l'articulation entre le pluralisme culturel et l'universel républicain, notamment à l'école. Elle vient de publier: Laurence De Cock et Régis Meyran (dir.), Paniques identitaires, identités et idéologies au prisme des sciences sociales, Le Croquant, 2017 et Laurence De Cock (dir.), La Fabrique scolaire de l'histoire II, Agone, 2017.

\begin{abstract}
A cteur multipositionnel, l'Association pour la connaissance de l'histoire $\mathrm{I}_{\text {de l'Afrique contemporaine (Achac) est une association de chercheurs et }}$ chercheuses sur le passé colonial née en 1989, et dont le but était de donner une visibilité à la mémoire coloniale dans la société française, en mobilisant des répertoires d'action variés. L'Achac a réussi à se bâtir une position d'incontournable pivot à la fois dans la détermination de diagnostic et dans la prestation de services en direction de différentes institutions et collectivités territoriales. Grâce à un équilibre toujours recherché entre le monde académique et le monde profane, l'association entretient son capital savant tout en maintenant un capital militant contre le racisme et pour une république multiculturelle. La transmission de l'histoire coloniale est au cœur de sa démarche. Ce faisant, l'Achac nourrit la corrélation entre la connaissance du passé colonial et le traitement du « problème de l'immigration » dans la société. L'angle proposé par l'Achac relève d'une sorte de thérapie mémorielle et repose sur l'idée qu'une meilleure transmission du passé colonial apaiserait la société en retraçant l'origine historique du racisme et en contribuant à une politique de la reconnaissance des populations héritières de l'immigration coloniale et
\end{abstract}


postcoloniale. Leur propos est fondé sur le postulat de la mise en place d'une « culture coloniale 1 » uniquement définie par le prisme des représentations des colonisés par les colonisateurs qu'ils appréhendent par l'inventaire et l'analyse de sources de propagande dont se dégage une multitude de stéréotypes coloniaux. Ce faisant, ils nourrissent la catégorisation de l'immigré par le prisme de son appartenance culturelle, en l'occurrence, descendant de colonisé. L'association s'inscrit donc dans la continuité du processus de traitement de la question de l'immigration depuis les années 1980, mis au jour notamment par Angéline Escafré-Dublet qui montre dans sa thèse comment l'histoire coloniale participe aussi d'une nouvelle manière de désigner les immigrés par le biais d'une « promotion culturelle » valorisant la culture d'origine ${ }^{2}$.

En ce sens, la stratégie de l'Achac participe d'une culturalisation de la question immigrée avec ceci de particulier que, par sa configuration, les acteurs qui y sont impliqués, ainsi que les actions mises en place, elle ajoute une dimension entrepreneuriale tendant à faire du passé colonial un véritable marché. C'est cet aspect que nous proposons d'interroger ici en retraçant la trajectoire de l'un des fondateurs, Pascal Blanchard, aujourd'hui responsable de l'Achac, ainsi que la stratégie de l'association s'apparentant à un marketing du passé pour lequel la catégorisation culturelle constitue un argument-clé. Ainsi, la démarche de l'Achac introduit une nouvelle coordonnée dans les usages sociaux du passé et de la mémoire qui jusque-là privilégiaient les circuits associatifs, familiaux ou politiques sans que n'intervienne de façon si ostensible la question de la rentabilité 3 .

\section{À l'origine de l'Achac, un entrepreneur bâtisseur : Pascal Blanchard}

Pascal Blanchard est un bâtisseur, du moins est-ce ainsi qu'il se présente. Lors d'un entretien d'une durée de deux heures trente, il nous fait part de son parcours professionnel assez atypique. L'entretien a lieu sur le site de l'Achac. Très vaste open space au cœur de Paris, le lieu est décoré de centaines d'objets et affiches de l'époque coloniale. Les bibliothèques sont remplies d'ouvrages publiés par l'association. Trois stagiaires ou employés travaillent sur de grands ordinateurs Macintosh. Le bureau de Pascal Blanchard est une pièce isolée, c'est là qu'il nous reçoit. L'entretien est très chaleureux avec tutoiement immédiat. Pascal Blanchard construit une connivence en prétendant « avoir lu tous [mes] écrits ». Issu d'une famille plutôt modeste, il a d'abord fait des études d'ingénieur:

1. Blanchard P. et al., Culture coloniale en France de la Révolution française à nos jours, Paris, CNRS éditions/Autrement, 2008.

2. Escafré-Dublet A., Culture et immigration : de la question sociale à l'enjen politique, 19582007, Rennes, Presses universitaires de Rennes, 2014.

3. Sur ces questions, voir la très récente synthèse de Gensburger S. et Lefranc S., À quoi servent les politiques mémorielles ?, Paris, Presses de Sciences Po, 2017. 
«Moi j’avais fait avant le génie civil... pour payer mes études, comme beaucoup d'étudiants, j'avais un métier qu'était constructeur de routes et de métro et je faisais la fac à côté. Donc j'ai fait d'abord mon génie civil, BTS machin, école d'ingénieur, enfin la classique, et en parallèle je faisais mes études d'histoire. Et c'est ça qui me permettait de me nourrir. J'allais construire le métro au canal de l'Ourcq la nuit. Et il y avait un gros avantage c'est que quand tu construis des tunnels, tu peux travailler en trois huit; et un gros avantage c'est que je faisais mes études la journée ; je faisais mes études d'histoire la journée et le métro du canal de l'Ourcq. J'avais deux métiers ${ }^{4}$ ».

Cette double casquette ${ }^{5}$ lui permet d'affirmer l'originalité de son positionnement dans le champ des historiens. C'est un registre qu'il adoptera régulièrement pour justifier aussi l'éventail de son répertoire d'action et sa multipositionnalité - «mon métier, c'est le béton et l'histoire » - lui permettant de mettre en avant le caractère entrepreneurial de sa démarche.

Pascal Blanchard fait des études d'histoire à la Sorbonne où il se lance dans une thèse sous la direction de l'historien africaniste Jean Devisse. Il y rencontre plusieurs camarades étudiants également inscrits avec ce directeur de thèse, dont Nicolas Bancel. Jean Devisse prévient ses doctorants qu'ils auront à batailler dur pour se faire accepter dans un champ académique peu ouvert aux recherches sur la colonisation. La thèse de Pascal Blanchard étudie les mutations du colonialisme dans le discours de la droite nationaliste des années 1930 au régime de Vichy en analysant la presse de l'époque ${ }^{6}$. Entre temps, lui et ses amis étudiants fondent une association en parallèle du travail conventionnel des séminaires et laboratoires de recherche :

«Voilà c'était un raisonnement qu'était très simple, c'est-à-dire soit on continue à pleurer comme font tous les africanistes le cul posé sur leurs chaises en disant "personne nous lit, personne s'intéresse à nos travaux", soit on fait l'inverse : comment on peut amener les gens à nos travaux ».

4. Entretien du 15 juin 2010. Sauf mention contraire, la plupart des citations qui suivent sont issues de notre entretien.

5. Il rappellera cet aspect de sa biographie lors d'un séminaire organisé à l'université de Paris 1 par l'équipe Humanitudes dans le cadre de l'atelier «valorisation de la thèse », le 16 février 2012. Son intervention est intitulée "Docteur en SHS, je crée ma boîte". http://calenda.org/207178., consulté le 25 mars 2016. À notre connaissance, il s'agit de la seule conférence en France de Pascal Blanchard sur cette question précise qu'il esquive généralement publiquement compte tenu de toutes les critiques que ces multiples activités suscitent dans le champ académique.

6. Blanchard P., Nationalisme et colonialisme: Idéologie coloniale, discours sur l'Afrique et les Africains de la Droite nationaliste française, des années 1930 à la Révolution nationale, thèse de doctorat de troisième cycle sous la direction de Jean Devisse, Université Paris-Sorbonne, 1994. 
L'idée de créer une association relève donc à la fois d'un désir de démocratiser la connaissance de l'histoire coloniale mais aussi de stratégies de carrière dans un champ apparemment négligé par la reconnaissance académique ${ }^{7}$. Tous choisissent alors de consacrer cette association à une thématique qu'ils estiment inexistante encore dans le champ : l'imaginaire colonial, à savoir l'ensemble des représentations du colon sur le colonisé, une idée que l'Achac élargira plus tard à la culture coloniale. Dans le cadre de l'entretien, Pascal Blanchard indique : «c'est l'imaginaire sur ce passé colonial qui dominait et qui faisait blocage ». Tous les membres voyagent beaucoup, sur le continent africain notamment, et glanent ici et là objets, cartes postales, affiches coloniales qu'ils stockent pour commencer à se constituer un fond. La narration de la genèse de l'association et de ses premiers travaux épouse une rhétorique de management : «Il fallait travailler l'opinion » nous indique-t-il.

"On était jeunes chercheurs, on était en maîtrise à l'époque, si on ne travaillait pas ensemble, on aurait du mal à faire écho à l'intérieur de l'université à nos travaux, trouver des moyens pour avancer notamment sur le corpus image et en même temps être en résonance vers l'externe au nom d'un principe... C'est parce que l'externe allait être demandeur de nos travaux qu'on allait avoir plus de facilités pour les mener à bien à l'interne. [Rires]. On devait être déjà très marketing sans le savoir 8 ».

C'est sur la thématique de la culture coloniale que les rejoint rapidement Sandrine Lemaire, alors doctorante, et dont les travaux portent sur l'Agence générale des colonies, une agence de propagande coloniale. Cette dernière, agrégée d'histoire, enseigne dans le secondaire et aura un rôle déterminant dans le travail de l'association en direction de l'école. L'équipe se stabilise ensuite autour du noyau dur constitué de Pascal Blanchard, Nicolas Bancel, Sandrine Lemaire et Emmanuelle Collignon ${ }^{9}$.

Le travail de l'équipe s'effectue dans plusieurs lieux et selon plusieurs modalités qu'il convient de décrire pour comprendre les formes et l'ampleur de la conquête du marché.

7. Une autre stratégie, adoptée par Nicolas Bancel par exemple, sera de quitter la discipline historique pour rejoindre les STAPS. Ayant fait sa thèse sur le sport à l'époque coloniale, cela lui permet d'obtenir un poste universitaire plus facilement.

8. Intervention de Pascal Blanchard, enregistrée lors du séminaire du 6 février 2012.

9. Emmanuelle Collignon a fait des études d'architecture. C'est la membre la plus discrète médiatiquement de l'Achac. Salariée par l'agence de Pascal Blanchard, les Bâtisseurs de mémoires (BDM), mais aussi vice-présidente de l'Achac, elle représente l'interface entre ces deux organisations. 
De fait, l’Achac a immédiatement orienté ses travaux vers des activités variées, savantes et profanes : séminaires, colloques, expositions, débats et publications 10 . Très rapidement, les tribunes médiatiques font partie de la stratégie de visibilité, de même que les publications semi-savantes et les rencontres/débats qui regroupent experts et citoyens, sorte de "forums hybrides 11 » c'est-à-dire des lieux destinés à accueillir des débats et à distribuer la parole entre spécialistes et non-spécialistes afin de démocratiser les savoirs et surtout de sortir de situations de crise.

Après avoir organisé une grande exposition à retentissement national intitulée « Images et colonies » en 1993, l'Achac se consacre davantage aux activités éditoriales à partir des années 2000. Impliquée dans les débats naissants sur la mémoire coloniale, elle multiplie tribunes, articles dans les revues savantes et semi-savantes (Hommes et migrations, Africultures), et publication d'ouvrages principalement collectifs. Le Monde diplomatique, Libération et Le Monde sont des supports privilégiés. La thématique est proche des préoccupations de Dominique Vidal, alors rédacteur en chef adjoint du Monde diplomatique. Sur le plan éditorial, l'Achac noue une relation particulière avec François Gèze, directeur des éditions La Découverte qui s’inscrivent dans le sillage des combats anticolonialistes des éditions Maspero. Les ouvrages mobilisent des universitaires dans une optique pluridisciplinaire et internationale, mais aussi des écrivains (Didier Daeninckx), des cinéastes (Rachid Bouchareb), des artistes (le taktikollectif de Toulouse) et des sportifs (Lilian Thuram).

" Tu vois ça [il me montre le coffret sur l'immigration dans les régions], c'est cent cinquante chercheurs, dix ans de boulot, trente iconographes, trois cent cinquante mille documents qui sont dans les archives ».

À partir de 2007-2008, l'association s'oriente à nouveau davantage vers l'organisation d'expositions. Un très long cycle est consacré à l'histoire régionale des immigrations, mais c'est surtout l'occasion de poursuivre la série d'expositions portant sur l'altérité dans le temps long et la fameuse notion de «zoos humains » lancée en 2000. Le « zoo humain » est une expression importée des recherches anglo-saxonnes par l'Achac. L'association la popularise en

10. Le site répertorie l'ensemble des activités de l'association depuis 1989. Pour un classement thématique et une analyse plus fine de l'évolution de ces activités, voir De Cock L., Le fait colonial à l'école : genèse et scolarisation d'un objet de débat public, scientifique et mémoriel (des années 1980 à 2015), essai de sociohistoire du curriculum, Thèse de doctorat en sciences de l'éducation sous la direction de Françoise Lantheaume, Université Lyon 2, 2016.

11. Callon M., Lascoumes P. et Barthe Y., Agir dans un monde incertain. Essai sur la démocratie technique, Paris, Seuil, 2001. 
travaillant notamment les moments d'expositions coloniales (1906, 1931) qu'ils analysent sous l'angle de l'exhibition à fondements racistes.

Cette diversification des activités relève d'une stratégie consciente. Il en va de même d'une autre activité de Pascal Blanchard, plus vertement commerciale celle-là, dans le cadre d'une entreprise dont il devient en 1996 l'un des codirigeants : les Bâtisseurs de mémoire (BDM). Cette agence propose aux entreprises d'organiser des expositions sur leur mémoire.

Une visite du site web de l'agence ${ }^{12}$ montre quelques analogies conceptuelles avec l'Achac. On y lit par exemple la volonté de cheminer de l' " histoire » à la « mémoire » à la «culture » de l'entreprise. Les accointances de Pascal Blanchard, que Camille Trabendi qualifie de "free lance researcher ", avec le monde du marketing agacent parfois ou interrogent pour le moins 13 car elles soulèvent la question de la rentabilité des opérations lancées par l'Achac, de ses partenariats et, de manière sous-jacente, questionnent le caractère désintéressé et neutre de ses productions savantes. Sur le blog des éditions Agone ${ }^{14}$, on trouve une critique très vive de son "postcolonial business ». Pascal Blanchard y est accusé de marchandiser le passé colonial afin de placer ses produits, et ce au détriment d'une certaine déontologie. L'agencement, aux contours flous, Achac-BDM place en effet le travail de l'association dans le champ économique et confère une autre dimension à son caractère entrepreneurial. La stratégie de «marketing ethnique » est d'ailleurs assumée et plébiscitée par Pascal Blanchard qui la vante dans quelques revues de marketing 15. Cette dimension entrepreneuriale se retrouve aussi dans l'une des activités de l'association qui s'est dotée d'une base de données de plusieurs dizaines de milliers d'images dont il explique l'origine ici lors d'une conférence à Paris 1 : « celui qui maîtrise les images, maîtrise tout », ajoutant, lors de notre entretien :

« Notre dernière idée de l'Achac, la meilleure idée qu'on ait eue, la meilleure, qui nous a donné une totale indépendance [... $99 \%$ du

12. http://www.lesbdm.com/accueil.html.

13. Trabendi C., «Pascal Blanchard en "free lance researcher" ", Agone, n 41-42, 2009, pp. 165193.

14. http://blog.agone.org/post/2010/05/18/Postcolonial-Business-1, consulté le 15 janvier 2017. L'auteur reproche, outre la mercantilisation des enjeux mémoriels, de travailler, dans le cadre des BDM, avec des entreprises compromises avec le passé esclavagiste comme Cointreau (rhum).

15. Le marketing ethnique réfléchit à des marchés spécifiques aux populations en fonction de leurs origines ethniques. L'Oréal ou Nivea sont les marques pionnières du champ. Pascal Blanchard a travaillé avec elles non seulement dans le cadre des BDM mais aussi au sein d'un projet scientifique sur le corps et la corporalité auprès de l'anthropologue Gilles Boetsch, collaborateur régulier de l'Achac. Nivea a cofinancé l'un des programmes de recherche. Voir le programme du colloque ici : http://www.achac.com/file_dynamic/programme-corps-etcouleurs.pdf, consulté le 29 septembre 2017. Sur le marketing ethnique, voir Blanchard P., "Le marketing ethnique est arrivé ! ", Africultures, 1er juillet 2003, article recensé sur le site de l'Achac. 
patrimoine était éparse. On est parti du postulat qu'il fallait qu'on constitue notre propre patrimoine. On allait dans des brocs, on achetait, on achetait, et ensuite on faisait des deals [...] Y a une époque ça ne valait rien. Dès qu'on finissait un programme, on vendait notre patrimoine, on gardait les droits, et on rachetait. Ce qui fait que l'Achac, depuis maintenant dix ans, réinvestit tous les ans à peu près 70000 euros dans le patrimoine iconographique. Dès qu'elle a une collection qui est constituée, on reproduit systématiquement les œuvres, on crée une base de données à partir de ça et, systématiquement, on vend à un musée ou à une institution publique nos collections. Aujourd'hui on doit avoir 20000 ou 30000 originaux et peut-être 100000,90000 documents ».

La médiatisation est également l'un des terrains d'action les plus importants de l'Achac. Dans les seules archives audiovisuelles, on compte quatorze passages de Pascal Blanchard entre 2005 et 2006. Cette présence agace beaucoup d'historiens du champ académique français. Pascal Blanchard évoque les lettres reçues par les rédactions des télévisions :

«Et là, le sommet que j'ai eu c'est un copain d'Arte qui m'a sorti toutes les lettres d'universitaires dénonçant qu'on passait trop à la télé. Et là tu lis avec grand plaisir, et je fais encore des sourires à tous ces gens-là que je connais très très bien ».

Vraie ou pas, l'anecdote témoigne d'un positionnement décalé mais assumé vis-à-vis du monde universitaire. Pascal Blanchard sait du reste reconvertir une faiblesse, le défaut de reconnaissance académique, en force : le positionnement économique et institutionnel.

\section{Les partenariats institutionnels}

L'ampleur de cette présence médiatique vaut enfin à l'Achac une reconnaissance institutionnelle importante. Des partenariats noués avec les collectivités territoriales permettent de financer les recherches et d'organiser des expositions ${ }^{16}$. À titre d'exemple, en 2006, l'Achac passe un accord avec la mairie du $12^{\mathrm{e}}$ arrondissement pour un programme commun de manifestations sur l'exposition de 1931 et y organise un grand débat-conférence où les piliers du groupe sont présents. De façon plus régulière, le Fonds d'aide et de soutien pour l'intégration et la lutte contre les discriminations (FASIDL) devenu l'Agence nationale pour la cohésion sociale et l'égalité des chances (ACSE) en

16. Ce soutien financier des collectivités régionales est aussi à l'origine des tensions entre le monde universitaire et l'Achac. Dans le cadre des projets de recherche sur l'immigration dans les régions, certaines universités engagées également dans des projets de recherche sur le même sujet ont pu être lésées de leur financement. 
2006 a cofinancé également des enquêtes et rapports. Cet appui institutionnel est un autre élément-clé du succès de l'Achac qui produit (et vend) des ressources (films, expositions, conférences) accessibles au grand public. Or, depuis les années 1990, les collectivités territoriales se lancent dans des projets de valorisation patrimoniale dans lesquels l'immigration tient une place de choix. C'est le cas de la région Midi-Pyrénées qui, en 2003, commande à l'Achac une enquête financée par la Division interministérielle de la ville (DIV) dans le cadre du programme « Mémoire, ville, intégration et lutte contre les discriminations ».

L'enquête dure six mois. Elle est menée par Pascal Blanchard, Sandrine Lemaire et Emmanuelle Collignon. Une synthèse des résultats est publiée sur le site du ministère de la Ville en janvier 2005, ainsi que dans l'ouvrage collectif La Fracture coloniale 17. Titré «Mémoire de l'immigration, mémoire coloniale, mémoire urbaine ", le rapport insiste sur la méconnaissance de l'histoire coloniale par les populations immigrées comme élément constitutif du malaise et $\mathrm{du}$ ressentiment éprouvé. Les enquêteurs préconisent alors quelques conseils et proposent la mise à disposition de ressources comme nous le verrons en détail par la suite.

Plus récemment, le Comité interministériel des villes a décidé le 18 février 2013, une nouvelle enquête sur la mémoire collective dans les quartiers populaires. Pascal Blanchard est membre et animateur du comité de pilotage « Histoire et mémoire des quartiers populaires 18 ». Le rapport est publié cette fois dans son intégralité en octobre 2013 après deux mois d'auditions en août et septembre. Intitulé « Histoires, patrimoine et mémoires dans les territoires de la politique de la ville », il propose en couverture « 15 recommandations de Pascal Blanchard 19 ». Sous le paradigme de la « diversité », le rapport insiste sur le pouvoir mobilisateur de la mémoire. Les habitants auraient perdu la mémoire de leurs quartiers qu'il faudrait donc valoriser. Les quinze recommandations touchent des domaines aussi variés que la constitution d'archives, le lancement de programmes de recherche, l'implantation d'entreprises, la création de spectacles vivants, films, expositions, etc. ${ }^{20}$. Il y est rappelé le poids de l'héritage colonial, à plusieurs reprises :

17. Blanchard P., Bancel N. (dir.), La Fracture coloniale, la société française au prisme de l'béritage colonial, Paris, La Découverte, 2005.

18. Le comité est restreint. On y trouve : Salah Amokrane, Tactikollectif; David Assouline, sénateur de Paris et historien; Pascal Blanchard, CNRS et Groupe de recherche ACHAC ; Pascale Boistard, députée de la Somme; André Bruston, expert ; Marc Cheb-Sun, journaliste ; Carole Da Silva et Mélanie Meharez, AFIP ; Régis Guyon, CNDP ; Pierre Lemonier, GPV d'Argenteuil ; Nanette Snoep, Musée du Quai Branly ; Naima Yahi, historienne de l'immigration.

19. Histoires, patrimoine et mémoire dans les territoires de la politique de la ville. Rapport au ministre délégué à la ville, François Lamy, recommandations, octobre 2013. http://i.ville.gouv.fr/index.php/reference/9313. Consulté le 29 septembre 2017

20. Ce rapport a provoqué des réactions très hostiles, notamment chez les historiens et sociologues des quartiers populaires qui dénoncent des contre-vérités et des conclusions hâtives 
«Aujourd'hui, on le constate, l'histoire d'inégalités issues du passé se prolonge sous des formes multiples dans le temps postcolonial » (p. 58).

En confiant ce rapport à Pascal Blanchard, les autorités, nationales cette fois, franchissent un cap dans la reconnaissance de la mémoire coloniale comme ressource patrimoniale et révèlent la place acquise par l'Achac dans le dispositif institutionnel de lutte contre les discriminations.

\section{L'usage de la culture coloniale et du continuum comme instruments de catégorisation culturelle}

Le continuum colonial est le fil rouge des travaux de l'Achac dès sa création. Il complète et explique la notion de culture coloniale fondée sur l'idée inspirée des postcolonial studies 21 - de la fabrication d'un imaginaire raciste par la situation coloniale (colonisation, moment colonial, décolonisation) qui perdure après la décolonisation et qui imprègne encore les sociétés contemporaines. L'expression continuum colonial soutient l'hypothèse que l'un des facteurs des discriminations au présent réside dans la relégation du passé colonial, voire sa non-prise en compte dans le récit national. Cette interprétation développe alors l'idée qu'une meilleure appréhension du passé colonial faciliterait l'intégration des populations héritières de l'immigration soucieuses de verser leurs héritages dans le pot commun historique.

Tous les travaux de l'association vont donc tourner autour de l'identification de cet imaginaire, à travers des sources quasi exclusivement de propagande (affiches de propagande, cartes postales coloniales, objets coloniaux), et la mise en avant (voire l'exposition) de la poursuite ininterrompue de ces représentations racialisées depuis la première colonisation du XVI ${ }^{\mathrm{e}}$ siècle. De tribunes en articles en ouvrages, les historiens de l'Achac déclinent tous les horizons ouverts par ces nouvelles sources qu'ils collectent, archivent et numérisent dans leurs locaux. Leurs travaux et interventions mettent au jour les non-dits républicains, les stéréotypes coloniaux et leurs linéaments contemporains. Dès 1997, un petit ouvrage de la collection «Découvertes » Gallimard présente à un public large l'évolution des représentations de l'indigène à l'immigré. C'est la première fois que le continuum est aussi clairement affiché. Au moment où la société française connaît les réminiscences et débats autour de la guerre d'Algérie et de la laïcité ${ }^{22}$, ces travaux ouvrent de nouvelles perspectives pour penser le racisme.

parfois proches du conflit d'intérêt. Voir Fourcaut A. et T. Tellier, « Les quartiers populaires vont-ils perdre la mémoire ? » Métropolitiques, 2014, http://www.metropolitiques.eu/Lesquartiers-populaires-vont-ils.html. Consulté le 29 septembre 2017.

21. Sur les postcolonial studies, cf. Smouts M.-C., La Situation postcoloniale. Les postcolonial studies dans le débat français, Paris, Presses de Science Po, 2007.

22. Voir Branche R., Guerre d'Algérie, la mémoire apaisée ?, Paris, Seuil, 2005. 
Les publications qui suivent approfondissent ce biais en interrogeant systématiquement la dimension ethnique des discriminations sur le temps long. Le miroir passé/présent est ouvertement brandi lorsqu'en 2000, au moment où se lance le programme sur les zoos humains, Pascal Blanchard accorde une interview au Monde. Les zoos humains sont l'occasion de critiquer la politique d'intégration:

"Quand on place un être humain dans un zoo, on affirme explicitement un discours de "différence". Et notre regard aujourd'hui sur les banlieues est-il si différent ? Quand ces lieux sont envisagés comme des "points noirs" selon M. Juppé, peuplés de sauvageons, d'après $\mathrm{M}$. Chevènement, ne reproduit-on pas les mêmes schémas, dans l'optique d'une nouvelle "mission civilisatrice" : l'intégration 23 ? 》.

L'idée de continuum est donc posée comme une ressource pour penser le politique.

Le moment le plus abouti de l'implantation de l'Achac dans le champ de l'histoire et de la mémoire coloniales est la publication en 2005 de La Fracture coloniale. Pascal Blanchard nous a relaté la genèse de cet ouvrage dont l'écho est considérable. Il est d'ailleurs réédité l'année suivante en poche. Il est entièrement placé sous le prisme du continuum colonial puisque certains articles traitent des banlieues et de l'immigration quand d'autres sont focalisés sur le passé colonial. L'origine de l'ouvrage remonte à l'enquête susmentionnée de 2003 faite à Toulouse. La DIV aurait d'abord refusé de rendre publique l'enquête.

"Donc comme on est cons et têtus, on s'est dit “merde fait chier ça fait un an qu'on bosse, et c'est beau notre boulot”. J'ai appelé Benjamin, j'ai appelé Lapeyronnie, j'ai appelé Boubeker puis tout le monde Françoise [Vergès]... Je dis “c'est nul ce qu'on a fait ?”. Ils disent "putain c'est vachement bon, attends c'est original”. Personne n'avait bossé là-dessus : le rapport entre l'état des savoirs, l'état des frustrations, les notions de fractures territoriales, culturelles, mémorielles, historiques, le ressenti... qu'un Pied-noir, qu'un Vietnamien qu'un Antillais qu'un Algérien ait à peu près le même ressenti, sentiment d'exclusion dans la société française face à l'histoire, personne n'avait bossé là-dessus en termes quanti. C'est con, alors tout le monde a dit “C'est bon, pourquoi tu fais pas un livre ?" [il se frappe les mains] Bonne idée, 2004 on se dit qu'on fait un bouquin ; je vais voir François Gèze, il me regarde avec des yeux, il me fait "la fracture coloniale ? Mais ça veut dire quoi ?" Je lui explique

23. «Notre regard sur les banlieues est-il si différent ? ", Le Monde, 16 janvier 2000. 
tatati tatata; donc on met déjà six mois à convaincre François qui est convaincu à un moment "Ben $\mathrm{OK}$ on va faire cinq cents exemplaires avec ton truc puis en plus tu veux publier ton rapport, t'es chiant, il fait six cents pages, tu réduis au moins les six cents pages” donc on fait une synthèse ; pour convaincre François faut quand même publier la synthèse, en bons chercheurs. On appelle les chercheurs et on arrive, on sort La fracture coloniale et... T'imagines. On est en septembre 2005. Ça fait déjà deux ans qu'on crapahute, on a commencé en 2002. En 2002 le lancement de l'étude DIV. 2003 on est sur le terrain. T'imagines le parcours ? Et on arrive en 2005, on sort notre truc ».

Dans le livre, l'article de Sandrine Lemaire est consacré à l'enseignement du fait colonial considéré comme partiel, lacunaire et n’opérant aucun lien avec la question de l'immigration. Cet article est le produit d'un long travail de fond à destination des curricula d'histoire.

\section{Le marché de l'histoire scolaire}

Très tôt, les historiens de l'Achac ont sollicité l'école, rouage essentiel de la démocratisation de leurs travaux. La première mention que nous avons trouvée remonte à 1996. L'Achac produit un Textes et documents pour la classe sur « L'Empire colonial à son apogée, propagandes et réalités 24 ». Deux ans plus tard, Pascal Blanchard, Nicolas Bancel et Sandrine Lemaire inaugurent un dossier spécial sur l'Afrique dans la revue Historiens et géographes 25 par l'article suivant : "L'Afrique noire inventée : de la Première Guerre mondiale aux indépendances ». Toutes les illustrations sont mentionnées comme issues de la collection privée de l'Achac : affiches de propagande. On touche là le mode d'entrée privilégié de l'Achac dans l'espace scolaire : l'offre de ressources pédagogiques.

Lors de notre entretien, Pascal Blanchard évoque à plusieurs reprises son souci de toucher les enseignants. Sandrine Lemaire, enseignante dans le secondaire, est décrite comme assurant ce rôle d'interface.

«Moi je suis pas très compétent là-dessus : ça a jamais été mon territoire ; moi j'avais qu'un seul truc qui m'intéressait c'est la partie illustrative des manuels parce que je pense que les manuels [inaudible] maintenant ça a un peu changé mais l'image coloniale c'était une

24. Textes et documents pour la classe est une publication de la très consensuelle maison d'édition du CNDP. Très utilisées par les enseignants, ces ressources, même si elles ne sont pas officielles, sont labellisées par le ministère de l’Éducation nationale qui y renvoie parfois dans son Bulletin officiel.

25. Afrique subsaharienne, Historiens et géographes, n 367, juillet-août 1999. 
catastrophe, c'est-à-dire qu'ils n'expliquaient jamais que c'était une image de propagande, disaient pas, c'était qu'illustratif ».

La stratégie a donc été, d'après lui, de développer un rapport de force avec les éditeurs. Comme il l'explique également dans sa conférence du 12 février 2012, l'Achac, grâce à son énorme stock d'iconographie, passe un «deal » avec les éditeurs scolaires : elle fournit gratuitement les images si les éditeurs les laissent les légender :

"C'est comme ça qu'on a fait changer beaucoup de choses et c'est Sandrine Lemaire qui a fait ça, parce qu'elle-même elle dirige beaucoup de manuels scolaires et on s'est rendu compte sur la question coloniale que les gens mettaient les images sans les questionner $26 \%$.

Dès le début des années 1990, avec l'exposition "Images et colonies ", l'Achac a développé cette stratégie en proposant des expositions itinérantes dans les établissements scolaires. La problématique scolaire intéresse très peu Pascal Blanchard lui-même, qui n'a d'ailleurs jamais enseigné, mais l'Éducation nationale est un marché. L'article de Sandrine Lemaire dans La Fracture coloniale est en quelque sorte l'aboutissement de cette conquête de marché. Pour le comprendre, il faut remonter à l'enquête de Toulouse en 2003. Dans la partie dite «quali » de l'enquête, trente-quatre personnes interrogées sont des élèves de lycée. Les enquêteurs s'étaient rendus sur place avec une mallette pédagogique pour soixante-huit participants.

\section{Contenu de la mallette pédagogique proposée par l'Achac}

La mallette se compose de deux éléments : un premier qui est donné aux sondés, un second qui reste à disposition pendant trois semaines.

Elle se structure en huit annexes :

1) Livres et revues, documentaires, films, CD-ROM (90\% sont des productions de I'Achac)

2) Invitations à des projections de films suivies d'un débat avec Sandrine Lemaire

3) Invitation à la conférence "De l'indigène à l'immigré »

4) Invitation à l'exposition «Images et colonies »

5) Vingt ouvrages récents sur la colonisation (deux seulement émanent du réseau Achac); vingt ouvrages récents sur l'immigration (seul un émane du réseau de l'Achac)

6) Dix-neuf articles photocopiés et remis à tous les participants (deux émanent du réseau Achac)

26. Conférence SHS susmentionnée 16 février 2012. Sandrine Lemaire a commencé en tant qu'auteure aux éditions Bréal en 1997. Elle prend la direction en 2010 du manuel de quatrième (comprenant donc l'histoire de la colonisation) chez Hachette. 
7) Vingt-cinq articles sur la colonisation remis à tous les participants (deux articles du réseau Achac dont la tribune réclamant un musée d'histoire coloniale)

8) Vingt-six articles sur divers thèmes remis à tous les participants (cinq émanent du réseau Achac)

Les résultats de l'enquête sont détaillés dans le rapport in extenso que nous avons pu nous procurer ${ }^{27}$. L'enquête prétend avoir choisi une ville neutre sur le plan de la mémoire coloniale ${ }^{28}$. L'analyse des questionnaires (aux questions fermées) montre une «défaillance des programmes scolaires qui accordent la portion congrue à cette page de l'histoire » (p. 10) ${ }^{29}$. Constatant par ailleurs de fortes interférences entre la mémoire familiale et la transmission scolaire, les auteurs en concluent qu'il est urgent d'intervenir à l'école, identifiant une forte demande sociale « formulée explicitement vis-à-vis de l'histoire coloniale» (en gras, p. 12). Enfin, ils signalent que les principaux schèmes coloniaux élaborés au temps de la colonisation ont conservé une certaine vitalité (p. 17), affirmant même que :

«Il ne fait guère de doute que la spectacularisation actuelle du débat sur l'insécurité et les banlieues - sans même parler du mot "jeune" qui opportunément remplace celui d'esclave, de sauvage, d'indigène, d'immigré ou de sauvageon - constitue une reformulation de ce que l'on pourrait appeler une fracture raciale » (p. 18).

Et, plus loin, que «l'identité de descendants de colonisés semble prendre corps en France, dans les espaces périurbains notamment » (p. 25). Beaucoup de jeunes sont encore pris dans une logique d'humiliation vis-à-vis de cette histoire, disent-ils, il y a donc un gros travail pédagogique à effectuer.

Le rapport fonctionne là encore sur le mode diagnostic/remédiation. La mallette permet de tester la pertinence du diagnostic de départ. Les enquêteurs indiquent que les usagers adultes ont massivement rejeté les articles spécialisés trop peu adaptés au grand public. Le livre De l'indigène à l'immigré est celui qui est accueilli le plus favorablement, voire avec enthousiasme (p. 249), grâce aux images, au format et au fait qu'il établit un lien direct entre les deux univers de la colonisation et de l'immigration. La moitié des sondés ont également utilisé Culture coloniale et Zoos bumains. Enfin, les auteurs constatent le

27. Blanchard B., Bancel N., Collignon E. et Lemaire S. (dir.), Enquête et étude, Mémoire coloniale, mémoire de l'immigration, mémoire urbaine, rapport à la délégation interministérielle à la ville, 2003. Rapport non publié in extenso. Il nous a été fourni par un acteur de la DIV.

28. C'est une affirmation très contestable du point de vue de l'histoire et de la réalité sociologique de la région Midi-Pyrénées et de la ville de Toulouse.

29. Une affirmation hâtive pour l'année 2003. La place du fait colonial dans les programmes est importante du primaire au lycée. Voir De Cock L., op. cit. 
grand succès de l'exposition (p. 241). Finalement, ils élaborent un classement pédagogique. L'exposition « est le support qui reçoit le satisfecit pédagogique le plus fort » (p. 254) et viennent ensuite les ouvrages. Les conférences, elles, sont peu plébiscitées. Au fond, la mallette pédagogique est présentée comme idéale pour réparer un mal-être identitaire, mis au jour par... la mallette.

Cette intense activité en direction de l'école porte partiellement ses fruits. En 2002 et 2003, l'ouvrage De l'indigène à l'immigré est indiqué en référence bibliographique des documents d'accompagnement des programmes de lycée ${ }^{30}$. Les problématiques des travaux de l'Achac sont présentes également dans les manuels scolaires entre les années 2003 et 2008. Les images sont attractives, nouvelles. Les affiches de propagande coloniale entrent dans les manuels scolaires. Cela épouse la logique commerciale de renouvellement iconographique de l'édition scolaire comme en témoignent les deux exemples cidessous :
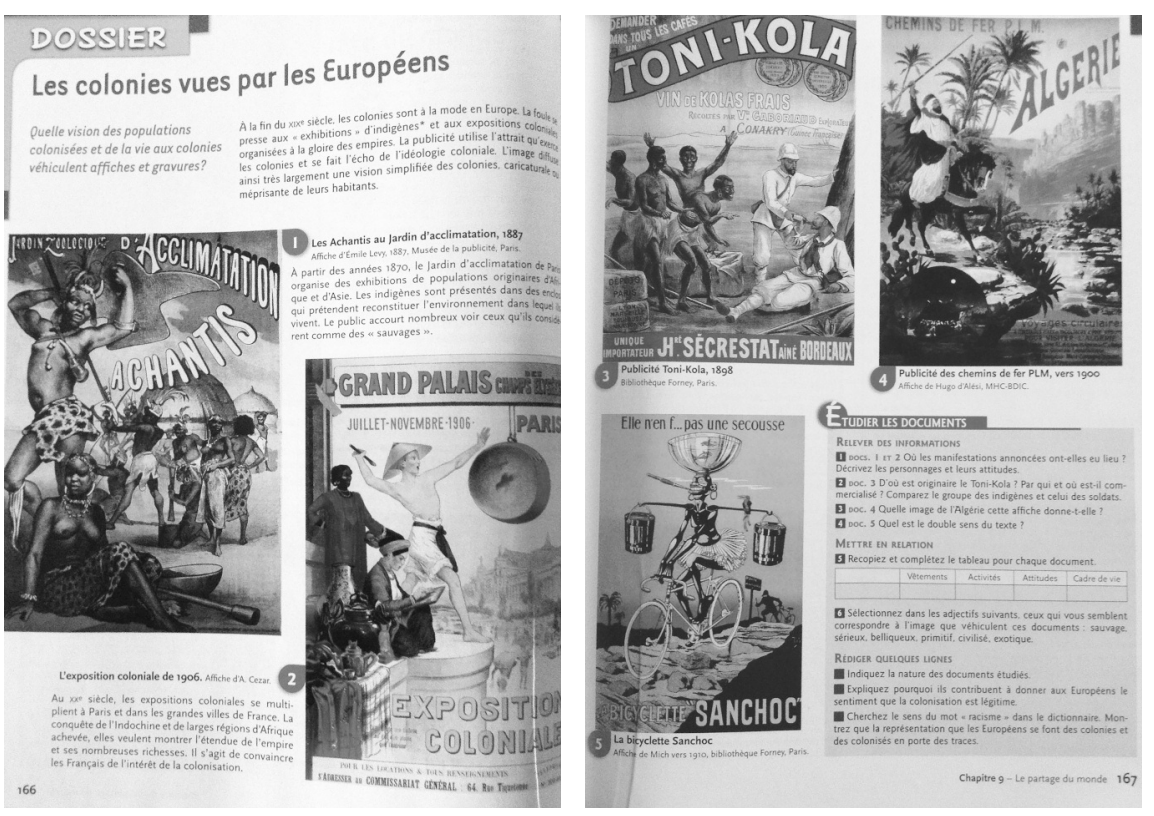

Double-page du manuel Baylac M.-H., Histoire-géographie, 4ème, Paris, Magnard, 2006, pp. 166-167 31

30. Ces documents ont un caractère semi-officiel, élaborés par des chargés de mission mais signés par l'Inspection générale. Publiés par le CNDP et visés par l'IGEN, ils sont porteurs d'une visée prescriptive même douce.

31. Au lycée, le manuel Hatier de 1ère L, ES, S consacre une double-page aux « représentations de l'indigène ", p. 74. Chez Bréal, le manuel de terminale consacre une double-page à "Comment les colonies sont-elles perçues par les Français dans l'entre-deux guerres ? ", p. 144. 
La double-page est montée par agencement d'affiches de propagande véhiculant les représentations des Européens sur les colonies et les indigènes, c'est-à-dire la culture coloniale. Ici toutefois, l'expression n'est pas prononcée. Le questionnement, et notamment le tableau, montre une volonté d'entrer très précisément dans l'identification de ces représentations par une description des détails de chacune des affiches. La question 6 - « sélectionnez dans les adjectifs suivants ceux qui vous semblent correspondre à l'image que véhiculent ces documents : sauvage, belliqueux, primitif, civilisé ou exotique » - fige l'analyse dans l'identification des stéréotypes.
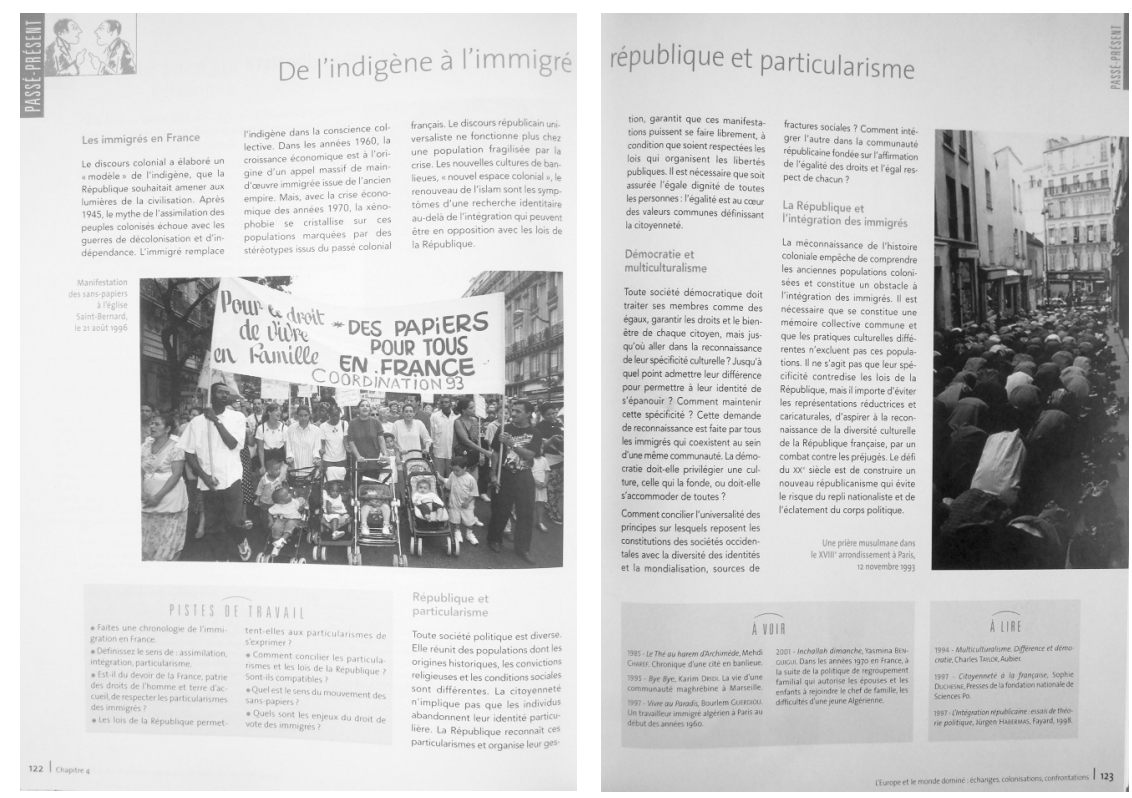

Le continuum colonial en classe de première :

Gaillard J.-M., Histoire, classe de première ES, L, S, Paris, Bréal, 2003

Ici la double-page assume la notion de continuum colonial. Intitulée « Passé-Présent », elle s'adresse à des élèves plus âgés et reprend la grille de lecture des documents d'accompagnement mentionnant l'ouvrage De l'indigène à l'immigré. Le récit reprend exactement les thématiques de l'ouvrage au point d'affirmer en page 2 que "la méconnaissance de l'histoire coloniale empêche de comprendre les anciennes populations colonisées et constitue un obstacle à l'intégration ». 


\section{Essentialisation et déshistoricisation de la question immigrée}

Les manuels rendent compte sans critique distanciée du fameux continuum, ce qui n'est pas sans poser problème. Sous couvert de « remettre de l'indigène » dans le récit scolaire, les supports documentaires (affiches de propagande, publicités coloniales) proposent un discours focalisé sur les stéréotypes coloniaux. Certes spectaculaires, ces documents ne permettent pas d'interroger les conditions de réception de l'époque, ni même d'ajuster les variables historiques de l'édification de cette « culture coloniale ». La démarche conduit à une forme d'essentialisation de la figure de l'indigène, au mépris des travaux universitaires qui insistent sur les phénomènes d'interactions, de rencontres, d'accommodements, de résistances, de métissage, etc. Le colonisé est enfermé dans son rôle de victime, une « histoire de fantômes délicieusement fascinante 32 ». Ce faisant, le risque existe de fabriquer des cadres d'intelligibilité rapides et artificiels des relégations actuelles qui ne sont vues que sous un angle culturaliste. Pour des adolescents en construction identitaire, cela peut nourrir une forme d'ethnicisation des rapports sociaux. C'est aussi ce que lui reproche en substance le politiste Jean-François Bayart dans son ouvrage au titre évocateur Les Études postcoloniales, un carnaval académique 33 ou dans quelques débats médiatisés. Pour lui, ces approches ne font qu'essentialiser et déshistoriciser le passé colonial en obérant à la fois sa complexité et ses multiples facettes géographiques, mais aussi en minimisant le passé précolonial de ces sociétés. Il y a là le risque de substituer à une nécessaire réflexion sur la domination, une pensée réductrice et vidée de sa dimension sociale au profit d'une vision purement culturelle.

Les expositions proposées par l'Achac reprennent aussi ce prisme. Au musée du Quai Branly en 2012, une exposition s’intitule «Exhibitions, l'invention du sauvage ». Pascal Blanchard en est l'un des commissaires scientifiques. Le circuit est conçu comme une ligne ininterrompue de regard stéréotypé posé sur l'Autre, du XVIe siècle à aujourd'hui. On y croise des représentations des Inuits, de Pocahontas, des cabinets de curiosité, des expositions coloniales, etc. Tous les petits films qui rythment l'exposition sont produits par l'Achac. Les textes sont écrits par Pascal Blanchard. L'exposition a été très visitée. Certains paraphes du livre d'or laissent paraître quelques traces de tristesse et colère : «Les gens sont des sauvages ! ", ou encore "En tant qu'indigènes d'une de vos colonies, nous sommes très heureux de pouvoir nous sentir exotiques encore une fois. Merci, très belle expo ! Indigènes d'Asie » et enfin «Comment ma fille pourra-t-elle imaginer qu'une centaine d'années avant sa naissance, une centaine d'années seulement, on exposait ses amis... 34 ».

32. Merle I., Sibeud E., " Histoire en marge ou histoire en marche ? La colonisation entre repentance et patrimonialisation ", in Crivello M., Garcia P., Offenstadt N. (dir.), Concurrence des passés. Usages politiques du passé dans la France contemporaine, Aix-en-Provence, Presses universitaires de Provence, 2006, pp. 245-255. 
Comment s'explique le succès de l'Achac ? L'Achac/BDM forme un conglomérat pénétré de la culture entrepreneuriale, militante et académique. Fort de ces trois propriétés, l'enrôlement de la sphère académique a été très réussi en prenant appui sur les ouvrages collectifs associant des universitaires, et en cumulant les capitaux symboliques des auteurs français et étrangers, et de l'éditeur (forte renommée de la Découverte), l'Achac a su faire valoir l'opportunité d'entrer dans son réseau : visibilité des travaux et valorisation d'une science engagée qui expliquent la constellation d'auteurs impliqués à ses côtés sans être toujours d'accord avec ses orientations. Par ailleurs, la connaissance $\mathrm{du}$ fonctionnement du "marché » et des modalités de communication, explique l'occupation du terrain médiatique ainsi que les multiples partenariats à vocation «évènementielle ». Ces activités variées se nourrissent ensuite respectivement et élargissent le réseau aux sphères artistiques, sportives, mais aussi politiques (liens avec les mairies, avec des personnalités de gauche liées aux luttes antiracistes ou à la mémoire de l'esclavage, comme Christiane Taubira, etc.). Cette forte visibilité explique la désignation de Pascal Blanchard comme ressource pour des projets plus institutionnels dont le milieu politique recherche également la publicisation, ce qui justifie sa mise en avant lors du rapport commandé par la DIV en 2013. La thèse du continuum colonial, pourtant si fréquemment décriée, s'est retrouvée dans quelques ressources pédagogiques dont certains manuels scolaires, chose étonnante au regard de l'emprise de la matrice nationale-républicaine dans l'école en France. Cette emprise multisectorielle de l'Achac nourrit encore aujourd'hui la culturalisation de la question de l'immigration et contribue à minimiser le facteur social de la relégation comme le montrent les diverses expertises sollicitées par la politique de la ville.

33. Bayart J.-F., Les Études postcoloniales, un carnaval académique, Paris, Karthala, 2010.

34. Photographies prises du livre d'or de l'exposition, avril 2012. 
